\section{Antibiotika rational einsetzen}

A ntibiotika-Resistenzen werden zunehmend $\mathrm{zu}$ einem globalen $\mathrm{Ge}$ sundheitsproblem. Die Weltgesundheitsorganisation WHO warnt, dass ein „postantibiotisches Zeitalter" eine sehr reale Gefahr des 21. Jahrhunderts sei [http://who.int/drugresistance/documents/surveillancereport/en/].

Ein wichtiger Beitrag, um Resistenzentwicklungen entgegenzuwirken, ist ein rationaler, gezielter Einsatz von Antibiotika. In den vergangenen Jahren wurden Strategien für ein strukturiertes Vorgehen, ein sogenanntes Antibiotic Stewardship (ABS) erarbeitet [AWMFRegister $\mathrm{Nr}$. 092/001]. Bei Neugeborenen, Kindern und Jugendlichen sind jedoch eine Reihe von Besonderheiten $\mathrm{zu}$ beachten. Eine Leitlinie zum ABS in der Pädiatrie sei daher in der Entwicklung, berichtete Prof. Dr. Johannes Hübner vom Dr. von Haunerschen Kinderspital München. Das ABS zielt darauf ab, die Auswahl und Dosierung von Antibiotika sowie die Applikationsart und Therapiedauer zu optimieren, um den Behandlungserfolg zu verbessern, die Toxizität zu minimieren und Resistenzentwicklungen zu vermeiden.

Die nützliche Rolle, die Bakterien für den menschlichen Organismus und die Gesundheit spielen, wird zunehmend deutlich, seit genetische Untersuchungen des Mikrobioms möglich geworden sind, so Hübner. Die Entwicklung des Mikrobioms beginnt mit der Geburt. „Einer der Störfaktoren für die Entwicklung des normalen, physiologischen Mikrobioms ist die Gabe von Antibiotika“, erklärte Hübner - ein Grund mehr für deren rationalen Einsatz.

Bei der Pharmakotherapie in der Neonatologie ist die spezielle Physiologie von Früh- und Neugeborenen zu beachten. Viele Substanzen sind für diese Altersgruppe nicht zugelassen. Die verabreichte Dosis wird häufig noch aus Empfehlungen für Erwachsene extrapoliert. Resorption, Distribution, Metabolismus und Exkretion von Antibiotika unterscheiden sich jedoch zum Teil deutlich. Basierend auf aktuellen pharmakokinetischen Untersuchungen wurden für zahlreiche Antibiotika neue Dosierungsempfehlungen für Kinder entwickelt [Pineda LC, Watt KM: Clin Perinatol 2015;42:167-76]. Bei einigen Substanzen wird zudem ein therapeutisches Drug Monitoring empfohlen, um optimale Dosierungen zu gewährleisten [Ritz N et al. Pediatr Infect Dis J 2016; 35:570-2]. „Korrekte Dosierungen, die größtmögliche Effektivität bei möglichst geringer Toxizität erzielen, sind ein wichtiger Baustein des Antibiotic Stewardship", sagte Hübner.

Angelika Bauer-Delto

Hübner J: Antimicrobial Stewardship - Neonatologie.

\section{Neuer Kurs „Antibiotic Stewardship in der Pädiatrie"}

Ein neues Fortbildungsangebot ist der Kurs "Antibiotic Stewardship in der Pädiatrie", der erstmalig vom 21.-23. Oktober 2016 in München stattfindet. Dieser Kurs sowie der Infektiologische Intensivkurs (iik) bilden zusammen ein Basismodul, das zur DGPI-Zertifizierung als „Antibiotika-beauftragter Arzt in der Pädiatrie" berechtigt.

Weitere Informationen unter: http://akademie-muenchen.de/ABS/

unter vier Jahren erkranken am häufigsten $(11,3 / 100.000)$. Eine NTM-Infektion ist wahrscheinlich, wenn ein einseitiger Lymphknoten mit einer Größe von $2 \mathrm{~cm}$ über mindestens drei Wochen persistiert, der Tuberkulin-Hauttest positiv ist und andere Infektionen ausgeschlossen werden können, erklärte Henneke. Eine Tuberkulose sollte mittels Röntgen-Thorax abgeklärt werden. Bestätigt wird die NTM-Infektion mittels Lymphknoten-Histologie in Ergänzung zu einer positiven Kultur oder PCR-Testung.

Zur Therapie der NTM-Lymphadenitis liegen wenige Daten vor, erklärte Henneke. In einer randomisierten pro- 